\title{
2-LOCAL LIE ISOMORPHISMS OF NEST ALGEBRAS
}

\author{
CHANGJing Li AND FANGYAN LU
}

Abstract. Let $\mathscr{N}$ and $\mathscr{M}$ be nests on a separable complex Hilbert space $\mathscr{H}$ of dimension greater than 2, and $\operatorname{Alg} \mathscr{N}$ and $\operatorname{Alg} \mathscr{M}$ be the associated nest algebras. We show that every additive 2-local Lie isomorphism $\Phi$ of $\operatorname{Alg} \mathscr{N}$ onto $\operatorname{Alg} \mathscr{M}$ has the form $\Phi=\phi+\tau$, where $\phi$ is an isomorphism or a negative of an anti-isomorphism of $\mathrm{Alg} \mathscr{N}$ onto $\mathrm{Alg} \mathscr{M}$, and $\tau$ is a linear map from $\mathrm{Alg} \mathscr{N}$ into $\mathbb{C I}$ vanishing on a sum of commutators.

Mathematics subject classification (2010): 16W10, 47L35.

Keywords and phrases: Lie isomorphism, 2-local Lie isomorphism, nest algebras.

\section{REFERENCES}

[1] S. Ayupov, K. Kudaybergenov and A. Alauadinov, 2-local derivations on algebras of locally measurable operators, Ann. Funct. Anal. 4 (2013) 110-117.

[2] K. I. Beidar, M. Brešar, M. A. Chebotar and W. S. Mardindale III, On Herstein's Lie map conjectures (I), Trans. Amer. Math. Soc. 353 (2001) 4235-4260.

[3] D. BenKoviČ AND D. EREMITA, Commuting traces and commutativity preserving maps on triangular algebras, J. Algebra. 280 (2004) 797-824.

[4] M. BREŠAR, Commuting traces of biadditive mappings, commutativity-preserving mappings and Lie mappings, Trans. Amer. Math. Soc. 335 (1993) 525-546.

[5] R. L. CRIST, Local derivations on operator algebras, J. Funct. Anal. 135 (1996) 76-92.

[6] K. R. Davidson, Nest Algebras, Pitman Research Notes in Mathematics, Vol. 191, Longman, London, New York (1988).

[7] J. A. ERdos, Operators of finite rank in nest algebras, J. London Math. Soc. 43 (1968) 391-397.

[8] D. Hadwin, J. Li, Local derivations and local automorphisms, J. Math. Anal. Appl. 290 (2004) $702-714$.

[9] J. HoU, X. ZHANG, Ring isomorphisms and linear or additive maps preserving zero products on nest algebras, Linear Algebra Appl. 387 (2004) 343-360.

[10] L. HUANG AND F. LU, 2-local Lie isomorphisms of operator algebras on Banch spaces, submited.

[11] W. Jing, Local derivations of reflexive algebras, Proc. Amer. Math. Soc. 125 (1997) 869-873.

[12] B. E. Johnson, Local derivations on $C^{*}$-algebras are derivations, Trans. Amer. Math. Soc. 353 (2000) 313-325.

[13] S. Kim And J. Kim, Local automorphisms and derivations on $M_{n}$, Proc. Amer. Math. Soc. 132 (2004) 1389-1392.

[14] D. R. Larson And A. R. Sourour, Local derivations and local automorphisms of $B(X)$, Proc. Sympos. Pure Math. 51 (1990) 187-194.

[15] Y. Lin And T. Wong, A note on 2-local maps, Proc. Edinb. Math. Soc. 49 (2006) 701-708.

[16] L. W. Marcoux And A. R. Sourour, Lie isomorphisms of nest algebras, J. Funct. Anal. 164 (1999) 163-180.

[17] W. S. Mardindale III, Lie isomorphisms of prime rings, Trans. Amer. Math. Soc. 142 (1969) $437-$ 455.

[18] C. R. Miers, Lie isomorphisms of factors, Trans. Amer. Math. Soc. 147 (1970) 55-63.

[19] L. Molnar, Local automorphisms of operator algebras on Banach space, Proc. Amer. Math. Soc. 131 (2003) 1867-1874.

[20] R. V. KADISON, Local derivations, J. Algebra. 130 (1990) 494-509.

[21] J. R. Ringrose, On some algebras of operators, Proc. Lond. Math. Soc. 15 (1965) 61-83. 
[22] J. R. Ringrose, On some algebras of operators II, Proc. London Math. Soc. 16 (1966) 385-402.

[23] P. ŠEmRL, Local automorphisms and derivations on B(H), Proc. Amer. Math. Soc. 125 (1997) 2677 2680. 